\title{
Comparative Study of Bit Error Rate with Channel Estimation in OFDM System for M-ary Different Modulation Techniques
}

\author{
Brijesh Kumar Patel \\ M. Tech. Scholar \\ Technocrats Institute of \\ Technology. \\ Bhopal
}

\author{
Jatin Agarwal \\ Professor \\ Technocrats Institute of \\ Technology. \\ Bhopal
}

\begin{abstract}
In this paper we investigate the bit error rate performance of orthogonal frequency division multiplexing system with channel estimation through least square error (LSE) and minimum mean square error (MMSE) algorithms. In this paper we are implementing the $\mathrm{M}$-ary phase shift keying (MPSK) and M-ary quadrature amplitude modulation (M-QAM) digital modulation techniques for mapping the signal in OFDM system for bit error rate evaluation with the employment of comb-type pilot symbol-assisted channel estimation algorithm over frequency selective multi-path Rayleigh fading channel. Here the performance of Least Squares method depends on not only on the signal-to-noise ratio (SNR) but also on the pilot ratio. Simulation results show that applying the LSE and MMSE channel estimation algorithm, they produce the best BER performance over the fading channel. We can consider the OFDM system as either a modulation technique or a multiplexing technique. In a single carrier system, a single fade or interferer can cause the entire link to fail, but in a multicarrier system, only a small percentage of the subcarriers will be affected [1]. Here we are using the pilot symbols as a guard band to eliminate the Inter Symbol Interference, and cyclic prefix eliminates the Inter Subcarrier Interference.
\end{abstract}

\section{Keywords}

Channel estimation, orthogonal frequency division multiplexing (OFDM), least square error (LSE), minimum mean square error (MMSE), pilot symbol, bit error rate (BER).

\section{INTRODUCTION}

In OFDM system, firstly we divide the available spectrum into subcarriers, and each subcarrier has a low rate data stream. The subcarriers have appropriate spacing and passband filter shape to satisfy orthogonality [1]. With the help of guard band we prevent the overlapping of subcarriers, in the OFDM system. OFDM can be considered as a spectrally efficient version of FDM [1]. The OFDM provides high data rate and low data loss in digital broadcasting and communications. OFDM provides good protection against cochannel interference and impulsive parasitic noise. Bit Error Rate is the rate at which errors occur in the transmission of digital data. The bit error rate or bit error ratio (BER) is the number of bit errors divided by the total number of transferred bits during a studied time interval. BER is a unit less quantity and expressed in percentage. The BER is affected by transmission channel noise, interference, distortion, bit synchronization problems, attenuation, wireless multipath fading etc. Here we are improving or reducing the bit error rate by using the different-different value of $\mathrm{M}$ (modulation alphabet) in the phase shift keying (PSK) and quadrature amplitude modulation (QAM) digital modulation schemes. In case of M-ary transmission we transmit two or more bits instead of one bit simultaneously. In the PSK modulation scheme we use a finite number of phases and in case of QAM finite number of at least two phases and at least two amplitudes are used. An in phase signal (I) and a quadrature phase signal (Q) are amplitude modulated with a finite number of amplitudes and then summed in the Quadrature Amplitude Modulation (QAM). Here the symbol consists of number of bits $(0 \& 1)$ and is represented by the particular phase, frequency, or amplitude. If modulation alphabet $\mathrm{M}=2^{\mathrm{N}}$, then this represents that each symbol represents a message consisting of $\mathrm{N}$ bits. We represent modulation alphabet (M) on a constellation diagram showing the amplitude of I signal at the $\mathrm{X}$-axis and the amplitude of Q signal at the $\mathrm{Y}$-axis for each symbol. Binary phase shift keying (BPSK) uses two phases, and quadrature phase shift keying (QPSK) uses four phases although any number of phases may be used. Additive White Gaussian Noise (AWGN) is used to mimic the effect of many random processes. AWGN is called 'Additive' because it is added to any noise that should be intrinsic. AWGN is called 'White' because it has uniform power across the frequency band for the information system. AWGN is called 'Gaussian' because it has a normal distribution in the time domain. In telecommunication we need multiplexing of signal means multiple analog or digital data signals are combined into one signal over a shared medium, and a reverse process known as de-multiplexing, with the help of de-multiplexing we can extract the original channels (signals) on the receiver side. Due to propagation media, attenuation and other effects the carrier is fade, and the fading may vary with time. In orthogonal frequency division multiplexing (OFDM) a signal is divided or split into several narrowband channels or subchannel or sub-carrier at different frequencies, in other words, we can say that OFDM is a method of digital modulation. OFDM provides high data rate transmission due to its flexibility and robustness against multipath fading [2]. Multipath channel fading exists when multiple delayed copies of a transmitted signal are received at the receiver, thereby resulting in the distortion of the transmitted signal [2]. These distortions are collectively termed 'channel fading', causing reception errors and hence increased bit error rate (BER) [2]. To reduce multipath fading and improve BER, different channel estimation algorithms are used to evaluate the distortion effect, noise, attenuation etc. In OFDM based systems, pilot-aided channel estimation usually involves some form of interpolation for deriving the estimates of channel distortions at non-pilot or data subcarrier locations within the 
symbol transmissions [2]. The BER performance not only depends on SNR, pilot pattern arrangement and interpolation method, but also on the channel model [2]. In this paper we consider an OFDM symbol block with $\mathrm{N}$ active subcarriers (data \& pilots), comprising $\mathrm{N}_{\mathrm{d}}$ data subcarriers and $\mathrm{N}_{\mathrm{p}}$ pilot subcarriers [2]. CP insertion creates a guard interval which prevents Inter-Symbol-Interference (ISI) between consecutive OFDM signals [2]. At the receiver of an OFDM system, the first step is the removal of CP samples, followed by the transformation of the remaining sequence into the frequency domain using the Fast Fourier Transform (FFT) [2]. The next stage is data recovery which involves channel estimation, to estimate the channel coefficients at the pilot subcarrier locations [2]. This is followed by interpolation, to estimate channel coefficients at data subcarrier locations [2]. Then channel equalization is implemented, to derive an estimate of the transmitted complex data-valued symbols [2]. The final stage involves signal de-mapping or demodulation, which maps the complex symbols to equivalent bit representation [2]. In this paper we are using block-type (insert pilots in the frequency domain) and comb-type (insert pilots in the time domain) pilot based channel estimation methods. Here we will estimate the channel at pilot frequencies with the help of LSE and MMSE method. In this paper our objective is to evaluate and improve the bit error rate (BER) performance of OFDM system by incorporating M-PSK and M-QAM digital modulation techniques over Rayleigh frequency selective multipath fading channel.

\section{LITERATURE SURVEY}

The concept of reducing bit error rate is not new. It was proposed from the invention of communication system but further more with the help of technology we are improving the BER and we are sending more data at a time with minimum loss. Past two decades technology made it faster, feasible and low time consuming. So many people proposed their concepts for improving BER in OFDM wireless communication system. Some of the relevant papers are discussed here:-

Firstly, K. Murali, M. Sucharitha, T. Jahnavi, N. Poornima, and P. Krishna Silpa described the novel design of time varying analysis of channel estimation methods in OFDM [1]. Three methods were performed to improve the BER in OFDM system. In the first method i.e. Least Square Error (LSE) is used to get the initial estimation. Second method i.e. Zero Forcing (ZF) algorithm is used to detect and separate the received signal. In the third method i.e. Minimum Mean Square Error (MMSE), the results confirmed the improvement of BER and high rate data stream [1]. The MMSE estimation yields much better performance than LS estimators, especially under the low signal to noise ratio (SNR) scenarios [1]. A major drawback of the MMSE estimator is its high computational complexity, especially if matrix inversions are needed each time the data in $X$ changes [1]. The result of channel estimation is affected by the signal to noise ratio (SNR) value. The larger the SNR the higher accuracy of the estimation will be. It relates to the selection of the channel estimation and detection techniques. The estimation results also affect the value of BER that would be decrease by increasing the value of SNR [1]. For high SNRs the LS estimator is both simple and adequate. However, for low SNRs, the presented modifications of the MMSE and LS estimators will allow a compromise between estimator complexity and performance. Even relatively low-complex modified estimators, however, perform significantly better than the LS estimator for a range of SNRs [1].
S. Adegbite, B.G. Stewart and S.G. McMeekin described the Least Squares Interpolation methods for LTE system channel estimation over extended ITU channels [2]. This paper investigates the bit-error-rate (BER) performance of linear, spline and piecewise cubic Hermite interpolating polynomial (PCHIP) interpolation methods in LS channel estimation over the three extended ITU channel profiles defined for Long Term Evolution (LTE) testing [2]. Simulation results show that applying the linear interpolation method produces the best BER performance over the fading channel with the smallest multipath delay spread. It is also shown that the choice of best interpolation method actually depends on the SNR in some of the fading channel profiles [2]. The extended ITU channel models; EPA, EVA and ETU that were considered in this paper were modelled in Matlab using the power-delay profiles [2]. The effect of the fading channel on the transmitted signals depends on the delay spread of the channel [2]. In this paper BER performance of LS channel estimation was calculated over single block transmissions of the OFDM symbol, assuming perfect synchronisation [2]. This paper shows that the linear interpolation method is the most appropriate scheme for all extended ITU channel models at zero doppler frequency and spline method gives the worst performance [2]. The possible suitability of linear interpolation in EPA could also be attributed to the much greater proportion of the number of pilots in relation to the delay spread [2]. This paper shows the performance of the interpolation methods in the EVA fading channel model at Doppler frequencies of 1.5, 15, 70 and $150 \mathrm{~Hz}$, the linear interpolation produced the worst performance while PCHIP gave the best performance [2]. In this case, the suitability of interpolation method does not only depend on the channel condition but also the SNR [2]. In this paper over the ETU fading channel, spline interpolation is the most suitable method while linear interpolation gives the worst performance [2]. This paper has focused on LS channel estimation in an LTE system for a standard pilot embedded OFDM LTE symbol over fading channel models that have been defined in LTE standards [2]. The simulation results show that linear interpolation is most suitable in LS channel estimation over both EPA and EVA fading channel models at zero Doppler frequency. At zero Doppler frequency in the ETU fading channel, linear interpolation is only suitable at lower SNR while PCHIP is most suitable at higher SNR [2]. In the EPA fading channel at non-zero Doppler frequencies upto $150 \mathrm{~Hz}$, linear interpolation is the most suitable method out of the three interpolation methods considered. However, in EVA fading, both PCHIP and spline interpolation methods, produce similar performance especially at lower SNR values. At higher SNR, PCHIP outperformed the spline interpolation method [2]. In the ETU fading channel, spline interpolation gave the best performance at non-zero Doppler frequencies at higher SNR [2]. The simulation results appear to suggest that the BER performance can be best optimised with a suitable interpolation method for a given channel fading condition and SNR. A form of Adaptive Interpolation Scheme (AIS) may therefore be appropriate towards achieving optimum performance for LS channel estimation [2].

\section{PROPOSED WORK}

\subsection{M-PSK and M-QAM}

To evaluate and improve the bit error rate (BER) in OFDM wireless communication system, so that M-ary phase shift keying (M-PSK) and M-ary quadrature amplitude modulation (M-QAM) in comb type (pilots insert in the time domain) pilot based Least Square Error (LSE) channel estimation algorithm over Rayleigh frequency selective multipath fading channel, becomes more effective to be used in communication 
applications. With the help of Least Square Error (LSE) algorithm we will evaluate the channel. Now we can write the transmitted and received signals as by [1],

$$
\begin{aligned}
& \mathrm{r} n=[\mathrm{r} \mathrm{n}, 0, \mathrm{r} \mathrm{n}, 1, \ldots \ldots . ., \mathrm{r} \mathrm{n}, \mathrm{k}-1] \\
& \mathrm{s} \mathrm{n}=[\mathrm{s} \mathrm{n}, 0, \mathrm{~s} \mathrm{n}, 1, \ldots \ldots ., \mathrm{s} \mathrm{n}, \mathrm{k}-1]
\end{aligned}
$$

These equations are in vector form. Here, $\mathrm{k}=0,1, \ldots \ldots \ldots . ., \mathrm{k}-1$ and $\mathrm{K}$ is the total number of subcarriers in an orthogonal frequency division multiplexing (OFDM) wireless communication system. Now, estimated channel frequency response

$$
\hat{\mathrm{H}} \mathrm{n}, \mathrm{k}=\mathrm{rn}, \mathrm{k} \div \mathrm{s} \mathrm{n}, \mathrm{k}, \quad \text { for } \mathrm{k}=0,1, \ldots ., \mathrm{K}-1
$$

The LS estimator minimizes the parameter $(\bar{Y}-\underline{X} \bar{H})^{\mathrm{H}}(\bar{Y}-$ $\mathrm{XH}$, where.Hmeans the conjugate transpose operation. It is shown that the LS estimator of $\bar{H}$ is given by .

$$
\hat{\mathrm{H}}_{\mathrm{LS}}=\underline{X}^{-1} \overline{\mathrm{Y}}=\left[\left(\mathrm{X}_{\mathrm{k}} / \mathrm{Y}_{\mathrm{k}}\right)\right]^{\mathrm{T}}
$$

Without using any knowledge of the statistics of the channels, the LS estimators are calculated with very low complexity, but they suffer from a high mean-square error. This channel estimation is based on a Gaussian channel model which provides better response because LSE estimator is low complex

\subsection{Block diagram}

The block diagram of the OFDM system with pilot-based channel estimation is shown in fig. 1. The system uses one modulator/de-modulator concept for transmission and receiving of binary data and to evaluate the bit error rate (BER) of OFDM wireless communication system. Firstly, the binary data grouped and then mapped into multi-amplitude multi-phase signals according to the type of modulation MPSK and M-QAM used in the modulator. After uniformly insertion of pilots between data, inverse fast fourier transform (IFFT) then transforms and multiplex the data sequence into time domain signal. After the IFFT block, we insert cyclic prefix (CP) and creates a guard interval in order to prevent inter-symbol interference (ISI) in OFDM wireless communication system. The transmitted signal is then convert parallel to serial and reached to a channel. At the receiver side, we add a noise into the transmitted signal called additive white gaussian noise (AWGN) and then signal will be serial to parallel. The guard interval (cyclic prefix) is removed and the received signals are sent to Fast Fourier Transform (FFT) block for de-multiplexing the received signal and transforming into the frequency domain. And now the pilot signals are extracted from de-multiplexed signals, after the channel estimation signal will be parallel to serially reached to de-modulator block and the signal will be de-modulated or demapped, and finally we got data in binary form on the receiver output. The transmitted binary data will be recovered with the knowledge of channel response by dividing the received signal to the channel response.

\section{RESULTS AND DISCUSSIONS}

Fig. 2 shows the MMSE/LS schematic view of previous work, implemented on Matlab, done by K. Murali, M. Sucharitha, T. Jahnavi, N. Poornima, and P. Krishna Silpa [1]. In this work Least Square Error (LSE) and Minimum Mean Square Error (MMSE) algorithms are used to time varying analysis of channel estimation methods in OFDM.

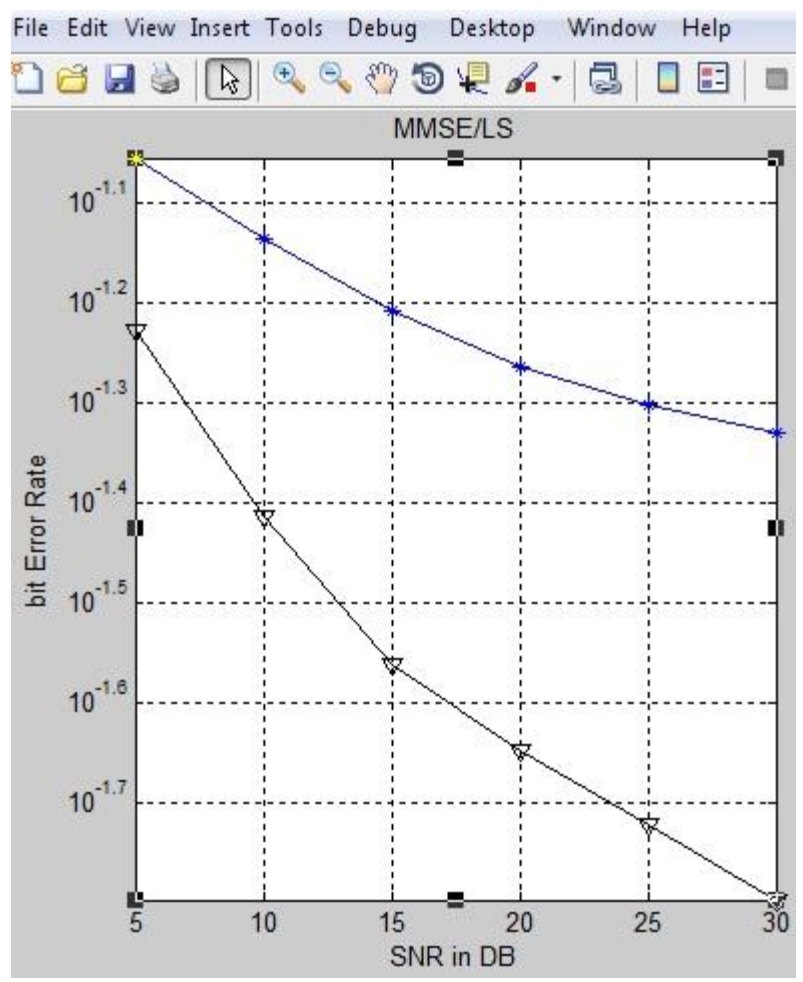

Fig 2: MMSE/LS graph for bit error rate of previous work, implemented in Matlab.

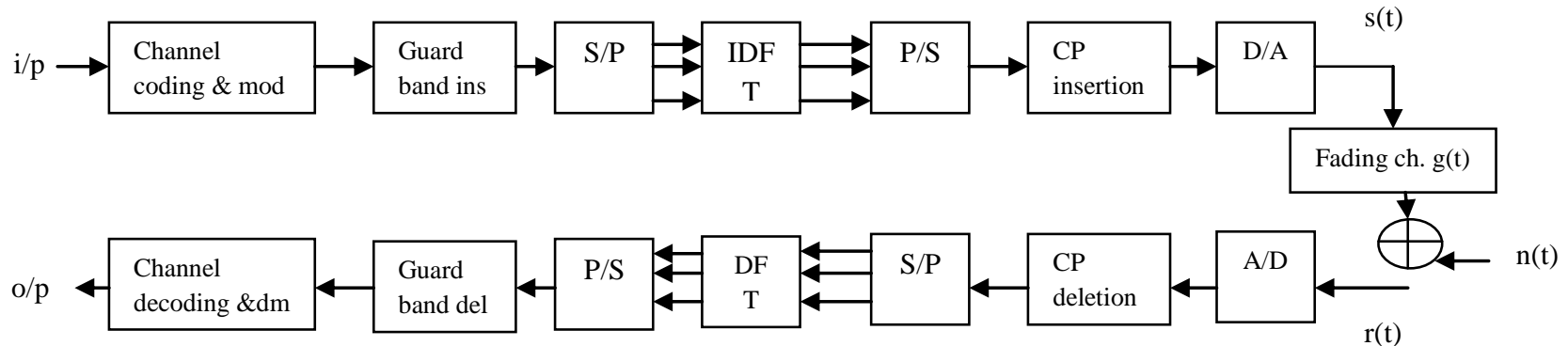

Fig 1: Basic OFDM system 
Table 1. Analysis of bit error rate based on previous LSE and MMSE algorithm

\begin{tabular}{|c|c|c|}
\hline $\begin{array}{c}\text { Type of } \\
\text { estimation }\end{array}$ & SNR (in dB ) & BER \\
\hline \multirow{7}{*}{ LSE } & 5 & 0.08477 \\
\cline { 2 - 3 } & 10 & 0.07336 \\
\cline { 2 - 3 } & 15 & 0.06195 \\
\cline { 2 - 3 } & 20 & 0.05458 \\
\hline \multirow{6}{*}{} & 25 & 0.04992 \\
\cline { 2 - 3 } & 30 & 0.04669 \\
\cline { 2 - 3 } & 10 & 0.05923 \\
\cline { 2 - 3 } & 15 & 0.03847 \\
\cline { 2 - 3 } & 20 & 0.02744 \\
\cline { 2 - 3 } & 25 & 0.01891 \\
\cline { 2 - 3 } & 30 & 0.01592 \\
\hline
\end{tabular}

Table 1 shows the bit error rate (BER) analysis with channel estimation in OFDM wireless communication system using Least Square Error (LSE) and Minimum Mean Square Error (MMSE) algorithm of previous work.

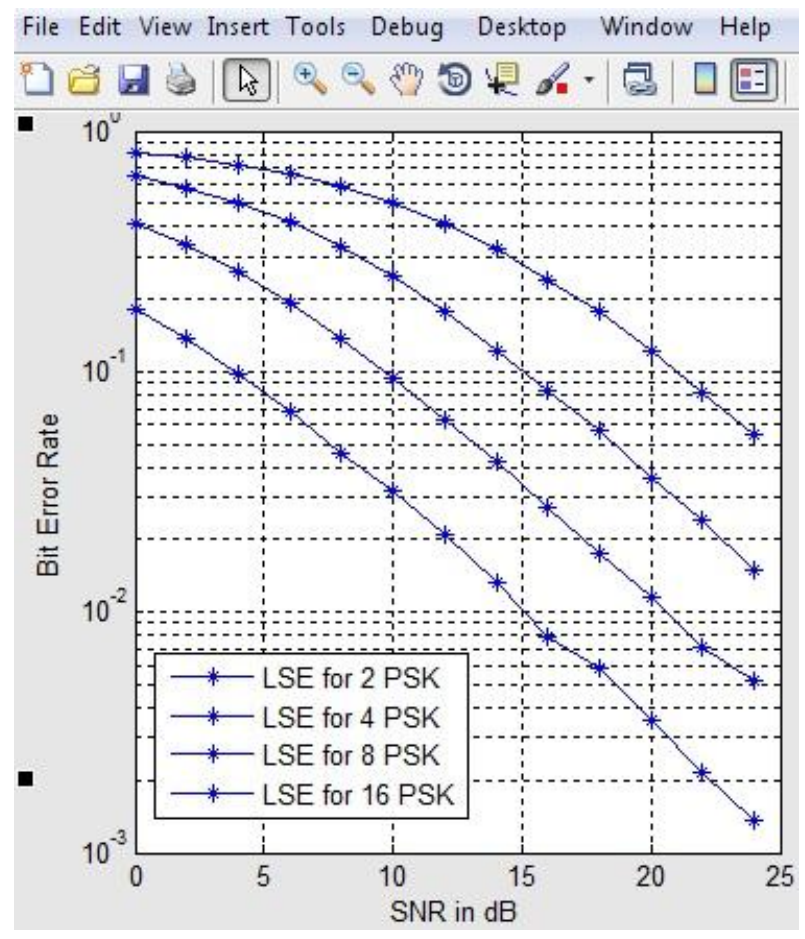

Fig 3: PSK in LSE graph for bit error rate of proposed work, implemented in Matlab.

Fig. 3 shows the Least Square Error (LSE) for Phase Shift Keying (PSK) schematic view of proposed work, implemented on Matlab. In this work, Phase Shift Keying (PSK) is used to evaluate and estimate the bit error rate with channel estimation at different value of $\mathrm{M}$ (modulation alphabet).
Table 2. Analysis of bit error rate based on proposed PSK in LSE algorithm

\begin{tabular}{|c|c|c|c|c|}
\hline \multirow{2}{*}{ SNR (In dB ) } & \multicolumn{4}{|c|}{ Bit Error Rate } \\
\cline { 2 - 5 } & 2 PSK & 4 PSK & 8 PSK & 16 PSK \\
\hline 0 & 0.1811 & 0.4126 & 0.6485 & 0.8123 \\
\hline 2 & 0.1383 & 0.3361 & 0.58 & 0.772 \\
\hline 4 & 0.09837 & 0.2575 & 0.5016 & 0.7226 \\
\hline 6 & 0.06871 & 0.1923 & 0.4194 & 0.6584 \\
\hline 8 & 0.04596 & 0.1383 & 0.3288 & 0.5845 \\
\hline 10 & 0.03217 & 0.0935 & 0.2502 & 0.4992 \\
\hline 12 & 0.02082 & 0.06298 & 0.1795 & 0.4104 \\
\hline 14 & 0.0132 & 0.04214 & 0.122 & 0.3219 \\
\hline 16 & 0.007879 & 0.02726 & 0.08407 & 0.2387 \\
\hline 18 & 0.005821 & 0.01759 & 0.05725 & 0.1761 \\
\hline 20 & 0.003549 & 0.01165 & 0.03626 & 0.1207 \\
\hline 22 & 0.002152 & 0.007192 & 0.02397 & 0.08167 \\
\hline 24 & 0.001353 & 0.005192 & 0.01498 & 0.05518 \\
\hline & & & & \\
\hline
\end{tabular}

Table 2 shows the bit error rate (BER) analysis with channel estimation in OFDM wireless communication system using Phase Shift keying (PSK) in Least Square Error (LSE) algorithm at different value of $\mathrm{M}$ (modulation alphabet) of proposed work.

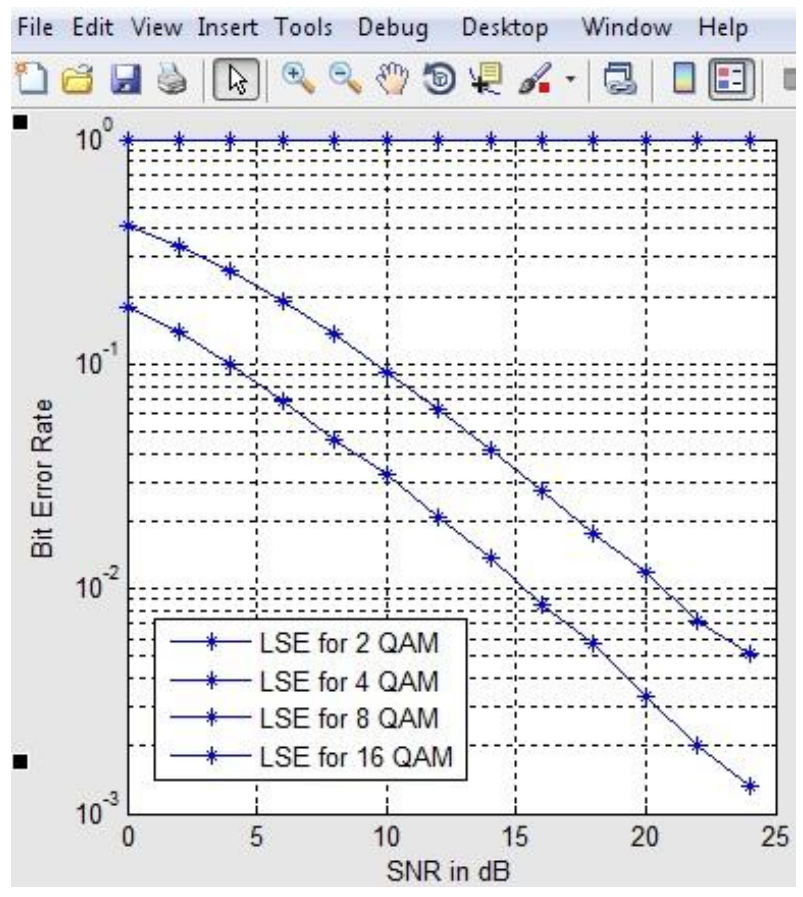

Fig 4: QAM in LSE graph for bit error rate of proposed work, implemented in Matlab.

Fig. 4 shows the Least Square Error (LSE) for Quadrature Amplitude Modulation (QAM) schematic view of proposed work, implemented on Matlab. In this work, Quadrature Amplitude Modulation (QAM) is used to evaluate and estimate the bit error rate with channel estimation at different value of $\mathrm{M}$ (modulation alphabet). 
Table 3. Analysis of bit error rate based on proposed QAM in LSE algorithm

\begin{tabular}{|c|c|c|c|}
\hline \multirow{2}{*}{ SNR (In dB ) } & \multicolumn{3}{|c|}{ Bit Error Rate } \\
\cline { 2 - 4 } & 2 QAM & 4 QAM & 16 QAM \\
\hline 0 & 0.1805 & 0.4148 & 0.9999 \\
\hline 2 & 0.1397 & 0.3348 & 0.9999 \\
\hline 4 & 0.0995 & 0.2594 & 0.9999 \\
\hline 6 & 0.06863 & 0.1913 & 1 \\
\hline 8 & 0.04639 & 0.137 & 1 \\
\hline 10 & 0.03213 & 0.09248 & 1 \\
\hline 12 & 0.02072 & 0.06362 & 1 \\
\hline 14 & 0.01369 & 0.04189 & 1 \\
\hline 16 & 0.008433 & 0.02714 & 1 \\
\hline 18 & 0.00567 & 0.01765 & 1 \\
\hline 20 & 0.003299 & 0.01182 & 1 \\
\hline 22 & 0.002031 & 0.007134 & 1 \\
\hline 24 & 0.001321 & 0.005129 & 1 \\
\hline
\end{tabular}

Table 3 shows the bit error rate (BER) analysis with channel estimation in OFDM wireless communication system using Quadrature Amplitude Modulation (QAM) in Least Square Error (LSE) algorithm at different value of $M$ (modulation alphabet) of proposed work.

Table 4. Simulation parameter

\begin{tabular}{|c|c|}
\hline Parameter & Specification \\
\hline FFT size & $\mathrm{N}=64,128,256,512$ \\
\hline Cyclic Prefix & $\mathrm{N} / 4$ \\
\hline Pilot interval & 8 \\
\hline Modulation & 2 \\
\hline Modulation types & M-QAM, M-PSK \\
\hline Channel length & 16 \\
\hline Number of iteration & 500 \\
\hline System bandwidth & $10 \mathrm{MHz}$ \\
\hline
\end{tabular}

\section{CONCLUSION}

After the analysis of proposed work and previous work from the bit error rate analysis table, we can conclude that if we will evaluate and estimate the bit error rate with channel estimation, the M-ary Phase Shift Keying (M-PSK) and M-ary Quadrature Amplitude Modulation (M-QAM) in Least Square Error (LSE) algorithm reduce more error as compared to LSE and MMSE algorithm and is an efficient solution for, transmission of digital data, establishing good communication and digital broadcasting and also provide high data rate for communication. The bit error rate as affected by the signal to noise ratio (SNR) value. As the SNR increases the bit error rate decreases but data rate increases, now we can say that larger the SNR value higher accuracy of estimation will be achieved. In other words we can say that bit error rate (BER) is inversely proportional to the signal to noise ratio (SNR), and SNR is directly proportional to the data rate. In future, we can do same simulation (M-PSK \& M-QAM) in Minimum Mean Square Error algorithm, so that we will transmit digital data with negligible or no loss of data with high data rate of transmission.

\section{REFERENCES}

[1] K. Murali, M. Sucharitha, T. Jahnavi, N. Poornima, P. Krishna Silpa, "A Novel Design of Time Varying Analysis of Channel Estimation Methods in OFDM," IJMIE, Volume 2,Issue 7,July2012.

[2] S. Adegbite, B.G. Stewart, S.G. McMeekin," Least Squares Interpolation Methods for LTE System Channel Estimation over Extended ITU Channels", International Journal of Information and Electronics Engineering, Volume 3,No. 4,July 2013.

[3] A. A. Quadeer and Muhammad S. Sohail, "Enhanced Channel Estimation Using Cyclic Prefix in MIMO STBC OFDM Sytems”, IEEE,2011

[4] Y. S. Cho, J. Kim, W. Y. Yang, and C. Kang, MIMOOFDM Wireless Communications with MATLAB, Singapore: IEEE Press, 2010.

[5] Fabien Delestre and Yichuang Sun, "A Channel Estimation Method for MIMO-OFDM Mobile WiMax Systems", IEEE, 2010

[6] I-Tai Lu and Kun-Ju Tsai, "Channel Estimation in a Proposed IEEE802.11n OFDM MIMO WLAN System", Sarnoff Symposium, IEEE, 2007

[7] M. K. Ozdemir and H. Arslan, "Channel estimation for wireless OFDM systems," IEEE Communications Surveys, vol. 9, pp. 18-48, 2007.

[8] F. Gao and A. Nallanathan. Blind channel estimation forofdm systems via a generalized pre coding. IEEE Trans. Vehicular Technol., 56(3):1155\{1164, May 2007.

[9] G. L. Stüber, J. R. Barry, S. W. McLaughlin, Y. Li, M. A. Ingram, and T. G. Pratt, "Broadband MIMO-OFDM wireless communications," Proceedings of the IEEE, vol. 92, no. 2, pp. 271-294, 2004. 